\title{
Echange d'informations médicales lors de rapatriements
}

\author{
Yvonne Gillia, Michelle Salathéb, Hans Wolffc
}

a Dr méd., membre du Comité central de la FMH, responsable du département Numérisation/eHealth; ' lic. iur., MAE, secrétaire générale adjointe de I'ASSM;

cProf., vice-président de la Conférence des médecins pénitentiaires suisses

1 www.sem.admin.ch

$\rightarrow$ Actualité $\rightarrow$ News

$\rightarrow$ News 2014

$\rightarrow$ Amélioration de

l'échange d'information

médicales lors des

rapatriements.

2 www.assm.ch

$\rightarrow$ Ethique $\rightarrow$ Médecine

pénitentiaire $\rightarrow$ Rapatrie-

ments sous contrainte.

La FMH, l'ASSM et la Conférence des médecins pénitentiaires suisses (CMPS) soutiennent une séparation claire entre les tâches du médecin traitant et celles du médecin accompagnant un rapatriement. Leur exigence est mise en œuvre grâce à de nouveaux formulaires: un rapport médical structuré et une liste de contre-indications pour les rapatriements par voie aérienne sont mis à la disposition du médecin traitant. Ce dernier communique la présence d'éventuels problèmes de santé - pour autant que la personne concernée le délie du secret professionnel - à son collègue en charge du vol de retour. Celui-ci se prononce sur l'aptitude au transport.

Depuis le décès tragique survenu en 2010 lors d'un rapatriement sous contrainte, le Secrétariat d'Etat aux migrations (SEM) s'assure que des médecins spécialistes et des professionnels de santé accompagnent les rapatriements sous contrainte sur des vols spéciaux et si nécessaire sur des vols de ligne. Cependant jusqu'ici, les tâches et les responsabilités de tous les acteurs n'avaient pas été définies de manière claire. En 2013, la Commission centrale d'éthique (CCE) de l'ASSM a publié une prise de position sur les aspects médicaux des renvois et exigé un changement de système (renoncement au «fit to fly»). La pression sur les médecins appelés à se prononcer sur l'aptitude au vol et le flux d'informations lacunaire entre les acteurs impliqués avaient suscité la critique. A l'initiative de la conseillère fédérale Simonetta Sommaruga, un dialogue a été mis en place en 2013 entre des représentants des autorités, les membres de la Commission nationale de prévention de la torture et des représentants du corps médical.

La FMH, l'ASSM et la Conférence des médecins pénitentiaires suisses (CMPS) ont participé à ce dialogue au nom des médecins. En septembre 2014, le dialogue a adopté en séance plénière un rapport ${ }^{1}$ sur les questions médicales qui se posent lors des renvois sous contrainte, dans lequel il présente son analyse et fixe les prochaines étapes. Il demande notamment la mise en œuvre de deux points fondamentaux:

1. Le rôle du médecin traitant avant le renvoi doit être clairement délimité par rapport au rôle du médecin qui délivre le certificat d'aptitude au renvoi.
2. L'indépendance du médecin à prendre une décision dans le respect du secret professionnel est primordiale pour la relation de confiance avec le patient. Elle est un préalable à l'amélioration de l'échange des données médicales.

\section{Changement de système de "fit to fly" à une liste de contre-indications}

La clarification des rôles telle qu'elle a été exigée est réalisée grâce à un changement de système passant d'un certificat d'aptitude au renvoi ("fit to fly") à une liste de contre-indications. Délivrer ce certificat étant une activité d'expert non conciliable avec le rôle du médecin traitant, ce dernier examine exclusivement la présence de contre-indications médicales et inscrit les éventuels problèmes de santé dans le rapport médical. En revanche, il n'évalue pas l'aptitude au transport, qui relève de la responsabilité du médecin chargé du suivi médical pendant le transport.

Pour la transmission d'informations médicales, le médecin traitant doit avoir l'accord du patient, et donc de la personne qui est sur le point d'être renvoyée, ou il peut se faire légalement lever du secret médical par les autorités concernées. En devenant concret, le changement de système apporte ici une solution dans le respect des principes éthiques fondamentaux ${ }^{2}$ de la médecine.

Afin d'accompagner ce changement de système, le SEM a mis sur pied un groupe de travail «Changement de système contre-indications» dans lequel ont siégé la 
FMH, l'ASSM et la CMPS aux côtés de la Confédération et des cantons. Sur les vols de ligne, il a procédé à une évaluation parallèle dès sa mise en place en avril 2015 et l'a poursuivie jusqu'au 30 septembre 2015 en se basant, de manière quantitative et qualitative, sur les retours des autorités cantonales en charge de la migration et des détentions, et du corps médical. Tous deux considèrent que ce changement de système est positif, tout comme la Commission nationale de prévention de la torture ${ }^{3}$ qui, dans le cadre d'un monitorage, observe tous les renvois de niveau 4 (décision de contrainte policière et mesures policières).

\section{Mise en œuvre définitive: nouveaux formulaires uniformes sur tout le territoire}

L'évaluation de septembre 2015 a permis au groupe de travail d'émettre des avis critiques et d'intégrer des propositions d'amélioration dans les processus en cours. En parallèle, les formulaires définitifs ont été validés et les différentes étapes précisées:
- Lorsqu'une décision de renvoi exécutoire a été prononcée, les autorités cantonales concernées s'assurent que les investigations médicales nécessaires dans le cadre de la préparation du départ aient lieu suffisamment tôt. Le médecin traitant est informé si l'entretien structuré fait état d'un danger pour la santé ou si la personne demande une évaluation médicale. Si c'est le cas, il rédige un rapport médical auquel est jointe une liste des contre-indications basée sur des références bibliographiques internationales.

- La liste des contre-indications ne remplace pas les évaluations minutieuses au cas par cas. Même les maladies qui ne remplissent pas strictement les critères de la liste peuvent exposer le patient à un danger inacceptable lors d'un rapatriement forcé. A titre d'exemple, la conjonction de plusieurs affections ou l'effet démultiplicateur du stress psychique lié à un rapatriement sous contrainte sur les symptômes peuvent être cités.

- Le médecin traitant établit son évaluation en toute indépendance et sa décision repose sur des principes
3 www.nkvf.admin.ch $\rightarrow$ Publications \& Services $\rightarrow$ Rapports

$\rightarrow$ Observations des renvois en vertu du droit des étrangers

$\rightarrow 2016 \rightarrow$ Rapport au Département fédéral de justice et police (DFJP) et à la Conférence des directrices et directeurs des départements cantonaux de justice et police (CCDJP) relatif au contrôle des renvois en application du droit des étrangers, d'avril 2015 à avril 2016.

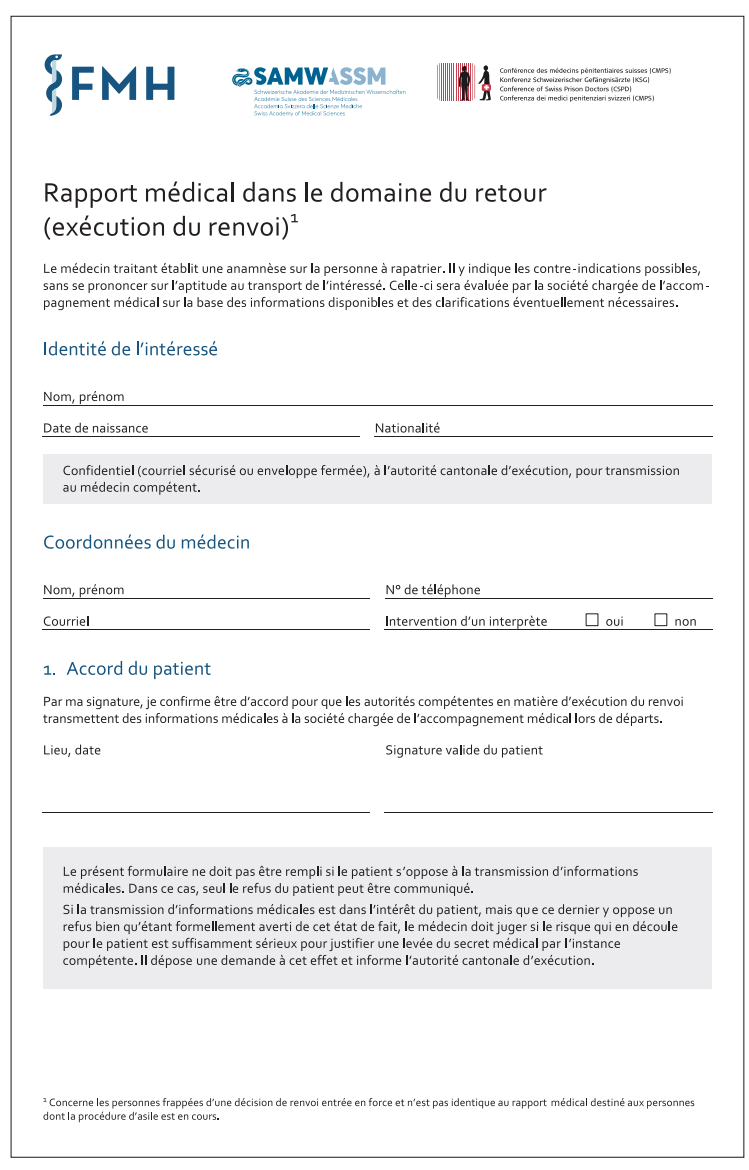

Figure 1: Le rapport médical pour le médecin traitant.

\section{Rapport médical dans le domaine du retour \\ 2. Durée du traitement}

Date d'examen

Date de début dutraitement

Douleurs et troubles annoncés

Problèmes de santé (y compris contre-indications constatées sur la base de la liste des contre-indications

3. Traitement et médication

Traitement actuel

Date de début

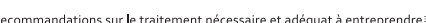

4. Remarques éventuelles du médecin

Date
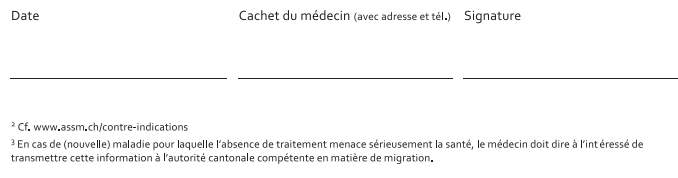
Correspondance:

FMH

Elfenstrasse 18

CH-3000 Berne 15

Tél. 0313591111

yvonne.gilli[at]fmh.ch

Académie suisse des

sciences médicales (ASSM)

Maison des Académies

Laupenstrasse 7

CH-3001 Berne

Tél. 0313069270

m.salathe[at]samw.ch

Conférence des médecins

pénitentiaires suisses

(CMPS)

Hôpitaux Universitaires

de Genève

Médecine pénitentiaire

Ch. du Petit-Bel-Air 2

CH-1224 Chêne-Bourg

Tél. 0223055218

Hans.Wolff[at]hcuge.ch

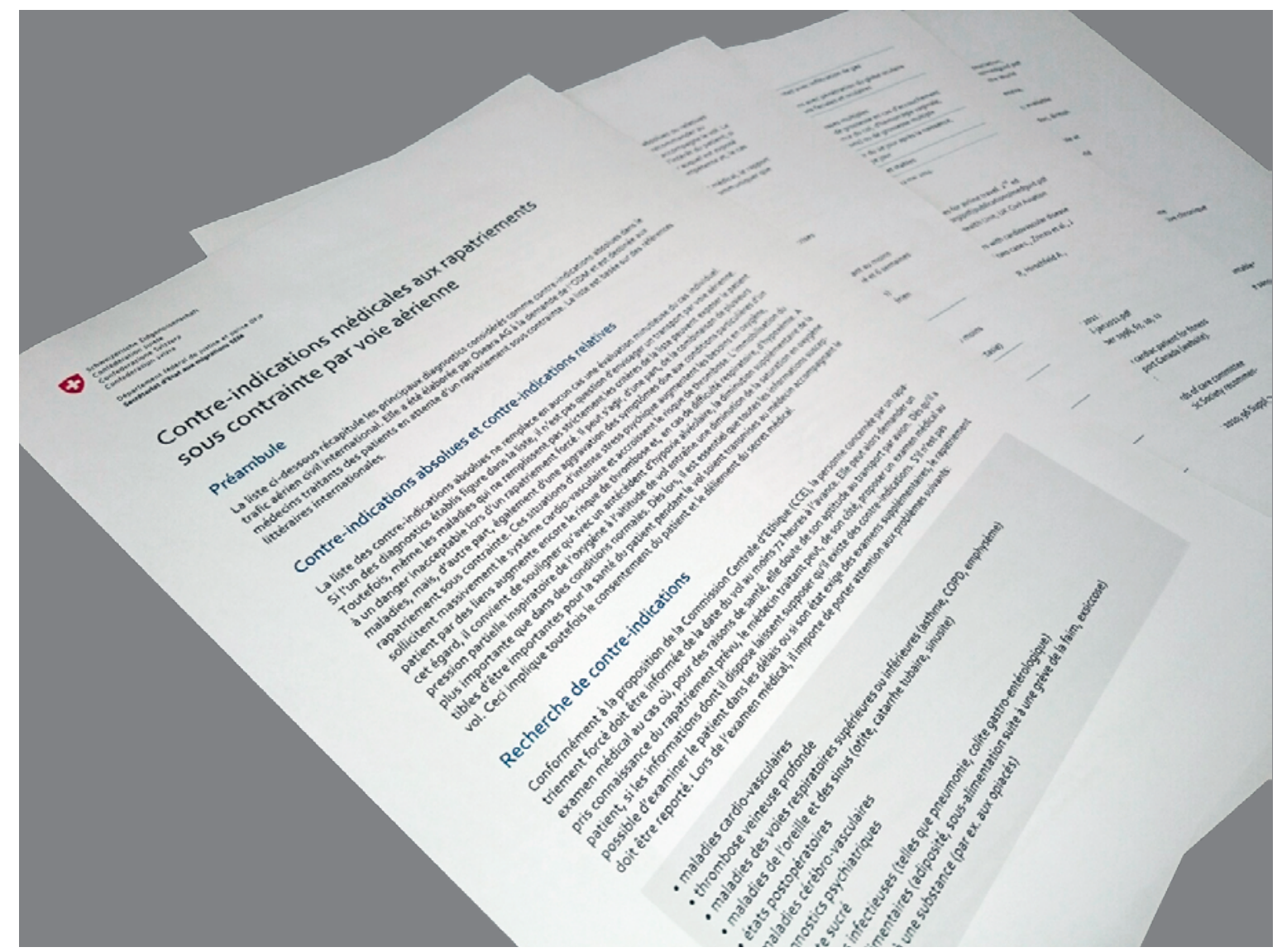

Figure 2: Une liste des contre-indications médicales aux rapatriements sous contrainte par voie aérienne est mise à la disposition des médecins.

médico-éthiques. Il peut proposer des examens supplémentaires si l'état de santé du patient l'exige. $\mathrm{Au}$ cas où le patient s'oppose à la transmission de ses données, le médecin a la possibilité de se faire lever du secret médical par les autorités cantonales. C'est à lui de lancer la procédure.

- En cas de contre-indications, le médecin traitant transmet son rapport de manière confidentielle au médecin chargé du transport. Pour que les données médicales puissent être transmises, la personne expulsée lève explicitement le médecin du secret professionnel.

Aussi bien le Comité central de la FMH que l'ASSM et la CMPS ont approuvé cette manière de procéder. 